\title{
Nouveaux risques dans les bas-fonds des terroirs soudaniens. Une étude de cas au Burkina Faso
}

\author{
Georges Serpantie $e^{1, *}$, Augustine Dorée ${ }^{2}$, Jean-Louis Fusillier ${ }^{3,4}$, Pascale Moity-Maizi ${ }^{5}$, Bruno Lidon ${ }^{3,4}$, \\ Manaka Douanio $^{6}$, Abdraime Sawadogo ${ }^{7}$, Aymar Yaovi Bossa ${ }^{8,9}$ et Jean Hounkpè $e^{8,9}$ \\ ${ }^{1}$ IRD, UMR GRED, Université Paul-Valéry, UMR GRED St Charles, route de Mende, 34199 Montpellier Cedex 5, France \\ 2 IRD, Montpellier Supagro, Montpellier, France \\ ${ }^{3}$ CIRAD, UMR G-EAU, Montpellier, France \\ ${ }^{4}$ G-EAU, CIRAD, Univ. Montpellier, Montpellier, France \\ 5 Montpellier Supagro, Montpellier, France \\ ${ }^{6}$ IRD, BP182, Ouagadougou, Burkina Faso \\ 7 IRD-CAP/M, Ouagadougou, Burkina Faso \\ ${ }^{8}$ West African Science Service Center on Climate Change and Adapted Land Use (WASCAL), 06 BP 9507, Ouagadougou, Burkina Faso \\ ${ }^{9}$ Institut national de 1'eau, Université d'Abomey-Calavi, 01 BP 526, Cotonou, Bénin
}

\begin{abstract}
Résumé - Dans la région soudanienne du Burkina Faso riche en bas-fonds, ces derniers représentent une facette du paysage inondable et fertile, ayant une fonction contre-aléatoire à travers des productions diversifiées en milieu humide en saison des pluies, et en contre-saison. Les activités de bas-fond permettent de réduire la vulnérabilité des groupes les plus exposés, comme les petites exploitations, les femmes et les jeunes, mais les arrangements fonciers restent précaires. C'est aussi un paysage particulièrement changeant avec des actions d'aménagement collectif hydraulique, rizicole et maraîcher. Les changements climatiques en cours atténuent l'aléa de la sécheresse sauf en début de saison, et un risque accru d'inondations est apparu. Ces tendances renforcent l'aléa du régime de l'eau, limitant les rendements, ce qui requiert des innovations dans les domaines technique et organisationnel, qui devront prendre en compte explicitement la dimension sociale et environnementale des bas-fonds.
\end{abstract}

Mots clés : plaine / risque / riz / jardin / adaptation

\begin{abstract}
New risks in Sudanian agricultural lowlands. A case study in Burkina Faso. In the southwest region of Burkina Faso rich in lowlands, these represent a facet of inundable, fertile landscape with an insurance function through production in a humid environment and in the off-season. Lowland activities help reducing the vulnerability of the most exposed groups, such as small farms, women and youth, but land tenure remains unstable. It is also a particularly changing landscape with collective hydraulic development actions to enhance rice and vegetable production. The current prevailing climatic change conditions mitigate the drought hazard except at the beginning of the season, and a new flooding risk has arisen. These trends exacerbate the vagaries of the water regime, limiting yields and requiring innovations in the technical and organizational aspects, taking into account social and environmental functions of lowlands.
\end{abstract}

Keywords: inland valley / risk / rice / market gardening / adaptation

\section{Introduction}

\subsection{Les bas-fonds et leurs aléas}

Les bas-fonds de la zone tropicale sont des fonds plats inondables de petites vallées situées en amont du réseau de

\footnotetext{
$\overline{* A u t e u r \text { de correspondance }}$ : georges.serpantie@ird.fr
}

drainage, emboîtés dans les épaisses altérations des socles cristallins (Raunet, 1985). Ils jouaient déjà un rôle antialéatoire et multifonctionnel dans les anciens terroirs (Lidon, 1993) et pourraient jouer un rôle croissant pour la sécurité alimentaire, à travers leurs potentialités en riziculture et pour faire face à d'éventuelles sécheresses (Sakané et al., 2011; Rodenburg et al., 2013). Les projets d'aménagement des basfonds ont cherché à provoquer une intensification de la 
riziculture, mais les pratiques actuelles en Afrique paraissent toujours persister à minimiser les risques dus aux contraintes et aléas éco-climatiques plutôt qu'à maximiser la production (Manzelli et al., 2015). Les contraintes principales sont la pression de l'enherbement (Moody, 1993), la fugacité de l'azote disponible (Yameogo, 2017), la toxicité ferreuse des terres hydromorphes (Audebert, 2006) et l'effet négatif de la submersion du riz à laquelle les variétés modernes sont particulièrement sensibles (Manzanilla et al., 2011). D'autres aléas sont apparus, comme la plante parasite Ramphicarpa fistulosa (Rodenburg et al., 2013), des difficultés d'organisation ou des conflits fonciers (Robin, 2018). S'il convient donc de poursuivre la caractérisation des nouveaux aléas et des impacts des aménagements sur ces derniers, gérer le risque dépasse évidemment la seule connaissance des aléas.

\subsection{La gestion du risque}

Le risque résulte de plusieurs composantes : les aléas, les enjeux et la vulnérabilité (Leone et al., 2010). "Gérer le risque », c'est donc évaluer l'aléa (pour mieux l'anticiper), agir sur ce dernier (atténuation), limiter les enjeux exposés (évitement des zones ou des périodes à aléas), réduire la vulnérabilité (s'assurer, capitaliser, répartir le risque, mutualiser les pertes, renforcer sa résilience).

Ces différentes dimensions en font un thème transdisciplinaire. Les agronomes et les géographes analysent les aléas du climat et comment les agriculteurs les perçoivent et prennent ou préviennent le risque en diversifiant les activités, les parcelles, les espèces et les variétés, en jouant à la fois sur l'intensif risqué et l'extensif rustique, en calant les cycles culturaux sur les périodes de moindre aléa ou en procédant à des actions de rattrapage (Eldin et Milleville, 1989). Les hydrauliciens cherchent comment atténuer les effets de la variabilité climatique à travers des aménagements (Lidon, 1993; Bossa, 2018). Les socio-économistes mesurent les impacts des aléas selon les vulnérabilités et les capacités de résilience des groupes sociaux (Blaikie et al., 1994; Lallau, 2008).

\subsection{Problématique}

Le Burkina Faso est particulièrement concerné par les aléas touchant les ressources en eaux et terres humides en tant que pays sahélien rural aux multiples vulnérabilités. Les zones humides comme les bas-fonds y ont joué un rôle agricole croissant à partir des sécheresses des années 1970 et 1980 (Serpantié et Zombre, 1994). L'aménagement des terres de bas-fonds pour la riziculture a été de plus en plus promu par les acteurs du développement en vue de sécuriser l'alimentation des villes (Jamin et Windmeijer, 1998; Drabo, 2004). Avec l'augmentation du prix du riz en 2008 sur le marché mondial, la promotion du riz national est même devenue une priorité vis-àvis d'autres céréales. La tendance est à l'expansion des aménagements vers les régions les plus humides, adaptées au riz mais moins concernées par les sécheresses. Il s'ensuit des tensions possibles entre des fonctions contradictoires : un rôle local multifonctionnel et anti-sécheresse exceptionnelle vs. les ambitions productives rizicoles nationales au nom de la sécurité alimentaire urbaine, mais aussi entre la recherche de sécurité (fonction anti-aléatoire) vs. les risques qu'y prennent les producteurs.

L'objectif de cet article est donc, d'une part, de comprendre les différents rôles que jouent aujourd'hui localement les basfonds (aménagés ou non) dans un contexte de changement global, notamment le rôle anti-risque local, et d'autre part, d'évaluer les nouveaux risques que les bas-fonds présentent du fait des changements locaux et globaux.

L'approche est systémique et contextualisée pour replacer la question du risque dans un ensemble d'éléments interagissant, historiquement et géographiquement constitué, le système agraire (Jouve et Tallec, 1994 ; Bainville, 2017).

\section{Contexte et méthodes}

Cette approche transdisciplinaire se focalise sur un cas d'étude: un territoire villageois. On y aborde les nouveaux aléas, la diversité des usagers des bas-fonds, leur perception des aléas, leurs structures et institutions, et leurs stratégies face au risque.

\subsection{Le terroir d'étude}

Le sud-ouest soudanien du Burkina Faso et ses projets d'aménagements innovants ont été sélectionnés comme cadre géographique adapté. La commune de Dano, chef-lieu de la province Ioba, est un territoire rural de langue Dagara, à population historiquement dense (Savonnet, 1976). Les nombreux bas-fonds y sont depuis longtemps dédiés à l'élevage, au riz et au maïs sur buttes (Pradeau, 1970; Pale et al., 2016). Puis, des projets ont introduit des aménagements dédiés à la riziculture: trains de diguettes en courbe de niveau pour rétention d'eau, ou systèmes de drainage entre des réseaux de casiers. La Fondation Dreyer y gère une filière rizicole intégrée d'achat, de transformation, d'organisation des producteurs, d'appui en mécanisation et d'aménagements basés sur le drainage.

Le climat soudanien de Boromo (station météo synoptique la plus proche de Dano) a reçu sur la période 1922-2013 une pluviométrie médiane de $940 \mathrm{~mm}$ (décennales : sèche $720 \mathrm{~mm}$, humide $1090 \mathrm{~mm}$ ).

Le village de Lofing $\left(11,1963^{\circ} \mathrm{N} ; 3,0486^{\circ} \mathrm{W}\right)$ se situe à $7 \mathrm{~km}$ de Dano. Son finage est estimé à $15 \mathrm{~km}^{2}$ pour 1650 habitants, soit une densité de $110 \mathrm{hab} / \mathrm{km}^{2}$. Les résidences sont organisées en quartiers, subdivisés en yirs (grandes « concessions » de plusieurs ménages). Lofing est établi autour d'un bas-fond représentant $7 \%$ de son finage.

\subsection{Méthodes}

Le dispositif de recherche adopté est multiscalaire. Les séries climatologiques décadaires (température, humidité, insolation, pluies et vents, ETP) de 1922 à 2013, de Boromo et Gaoua, encadrant Dano, sont analysées fréquentiellement, par la méthode des quartiles, des fréquences cumulées et du bilan climatique de Franquin (1984) en vue d'évaluer les aléas saisonniers du climat régional et leurs changements pluridécennaux, sur les paramètres pertinents pour l'agriculture.

À l'échelle du terroir villageois, des levés sont menés par parcours au sol et photo-interprétation d'images Google Earth 
a. Années déficitaires Boromo (quartile inférieur $\mathrm{P}<828 \mathrm{~mm}$ )

$$
\begin{aligned}
& \text { | | | | | | | | | || || || || | | | | | | | | }
\end{aligned}
$$

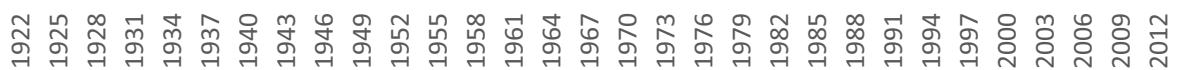

b. Années excédentaires Boromo (quartile superieur P>1032mm)

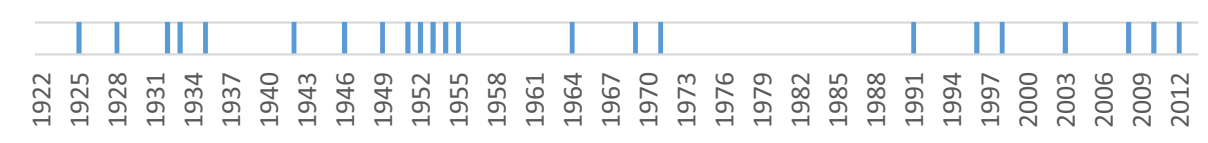

Fig. 1. Distribution des aléas annuels (Boromo, 1922-2013).

Fig. 1. Annual climate hazards distribution (Boromo, 1922-2013).

(logiciel Q-Gis) : terroir, paysages du bas-fond et de segments de bas-fond, entretiens qualitatifs auprès de personnes ressources sur les transformations passées et actuelles du système agraire (ressources, usages, activités, institutions...).

À l'échelle du bas-fond, nous avons procédé à 3 prélèvements de sol pour analyse. Nous avons suivi l'ensemble du régime de l'eau : la pluie (pluviographe à augets), les crues (limnigraphe à pression) et la nappe (piézomètres manuels relevés hors crues quotidiennement en interne [profondeur de l'eau dans le tube, représentant la pression de la nappe] et en externe [lame d'eau en surface]) sur deux années climatiques contrastées : 2017 , année de pluviométrie médiane à périodes sèches et 2018, décennale humide à crues.

À l'échelle de l'exploitation (18 unités), une enquête sur la diversité des systèmes de production a permis de connaître les structures d'exploitation et les pratiques, la façon dont les moyens de production sont mobilisés (notamment le travail agricole collectif, essentiellement de mars à novembre), la façon dont sont utilisées les récoltes, et la façon dont les risques sont perçus et traités. Ces enquêtes mènent à une typologie de structure, à partir d'un tableau de données structurelles traité par analyse multivariée ACP, puis classification ascendante hiérarchique.

Enfin, un réseau de suivi de parcelles de riz (26 en 2017, 54 en 2018), sur des sections de bas-fonds aménagées ou non, a été échantillonné par stratification en vue de disposer de données sur les pratiques, sur les coûts de production, et sur les variations de rendement en fonction des années, des aménagements et des pratiques (tests statistiques T sous Xlstat). Le rendement a été mesuré dans toutes ses composantes par échantillonnage de 1 à 5 placettes de $2,5 \mathrm{~m}^{2}$ environ (3 lignes de $3 \mathrm{~m}$ ) échantillonnées en vue de représenter la parcelle.

\section{Résultats}

\subsection{Les aléas pédo-hydro-climatiques et leurs impacts}

Les sols hydromorphes du bas-fond sont les plus fertiles du terroir : composés d'environ $50 \%$ d'argile gonflante, riches en limons et matière organique, ils ont une bonne activité de fissuration et de rétention d'eau, et une bonne capacité d'échange. Yameogo (2017) décrit dans d'autres bas-fonds de la zone un $\mathrm{pH}$ peu acide et une fugacité de l'azote disponible.
Ils sont sujets à des phénomènes de toxicité ferreuse par endroits (eaux rougeâtres, symptômes foliaires). Ils contrastent avec les pentes cultivées aux sols ferrugineux limono-sableux ou gravillonnaires pauvres, séchants et sensibles au ruissellement.

$\mathrm{Au}$ plan thermo-radiatif, la température à Boromo s'est accrue de près de $+2{ }^{\circ} \mathrm{C}$ en 50 ans pendant les mois secs et chauds (mars à juin) et de $+1^{\circ} \mathrm{C}$ en saison humide (juillet à octobre), représentant une difficulté accrue pour le travail manuel. La période froide de janvier est devenue plus fraîche $\left(-2{ }^{\circ} \mathrm{C}\right.$ à Gaoua, $0^{\circ}$ à Boromo, soit $-1{ }^{\circ} \mathrm{C}$ à Dano), une opportunité pour certaines productions maraîchères sensibles à la chaleur comme la pomme de terre, encore absente de cette région.

La distribution des pluviométries extrêmes sèches et humides annuelles (Fig. 1) permet de distinguer trois périodes : « historique» (1922-1970), «sèche» (décennies 1970-1980) et «actuelle» (après 1990). La période sèche a connu une succession de sécheresses particulièrement dommageables, souvent deux années consécutives. Depuis, le risque de sécheresse reste maintenu, mais atténué (une année sur trois). En revanche, le risque d'années excédentaires, porteuses d'inondation des bas-fonds, qui avait disparu de la période sèche, est revenu (une année sur trois).

L'analyse fréquentielle décadaire conduite sur ces trois périodes ne montre pas de variations significatives sur les dates de début, de maximum ou de fin des pluies.

En revanche, certains paramètres saisonniers montrent des variations ayant un impact sur la riziculture de bas-fond. En saison pré-humide (mai-juin), les décades favorables au semis sont celles dont la pluie est supérieure à ETP/2 (évaporation du sol nu) pour la recharge du sol en eau. Le piochage des sols argileux demande aussi des pluies suffisantes en mai ( $P>\mathrm{ETP} / 4)$. Or, la distribution statistique des pluies décadaires a changé significativement après la période sèche 1971-1990 (test Kolmogorov-Smirnov, au risque $5 \%$ ) (Fig. 2a). La fréquence de décades très sèches $(P<\mathrm{ETP} / 4)$ a presque doublé, impliquant une difficulté accrue d'installation précoce des cultures et une difficulté pour le piochage, réalisé sur une terre dure sous une chaleur croissante. Ces difficultés grandissantes sont confirmées par les paysans et particulièrement par les femmes.

Les crues précoces (juin-juillet) retardent les opérations rizicoles, abîment les sols labourés et les aménagements, et 


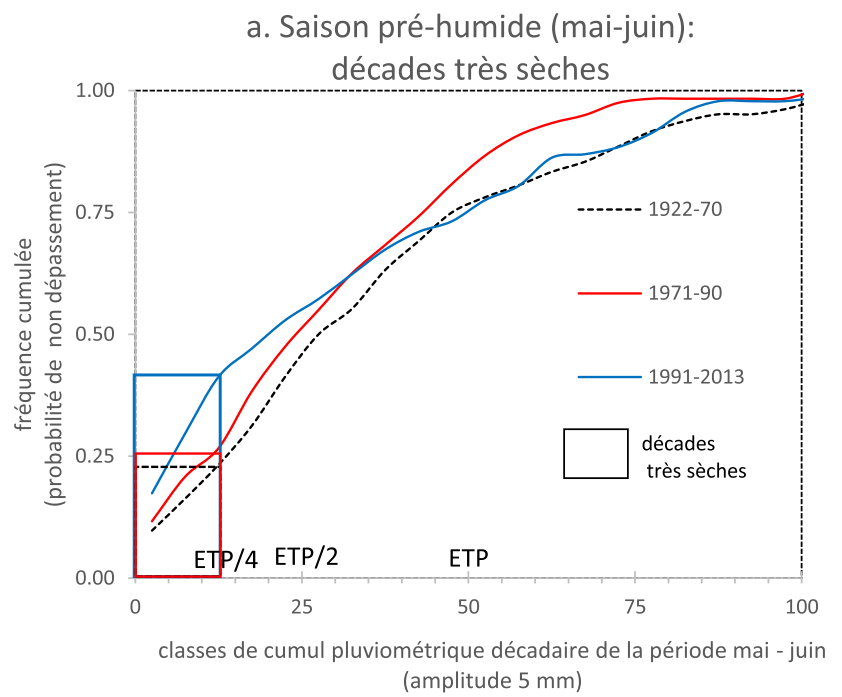

b. Saison des crues dommageables (juin-juillet)

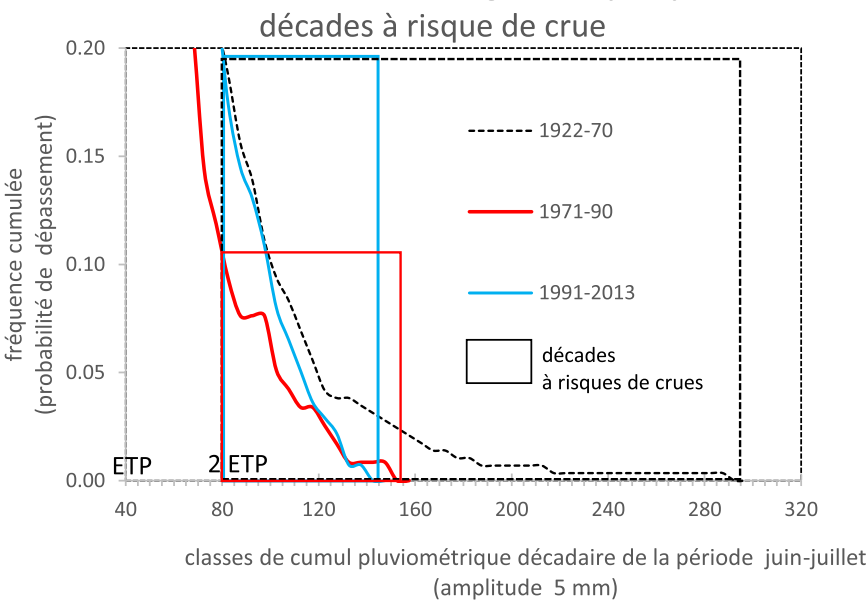

Fig. 2. Analyse fréquentielle des cumuls pluviométriques décadaires de la période pré-humide (Boromo). Décades sèches (2a) et décades à risque de crue $(2 b)$.

Fig. 2. Frequency analysis of decadal rains on the pre-humid season (Boromo).

submergent les jeunes cultures. Le risque de pluies de plus de $100 \mathrm{~mm} / \mathrm{j}$, générateur de fortes crues, inexistant pendant la période sèche, est apparu depuis (une année sur trois). Les crues se produisent aussi sous des pluies moindres, en cas de cumul antérieur de pluie élevé. Un cumul pluviométrique décadaire peut donc servir d'indicateur de l'aléa de crue en fin de décade. La figure 3, établie à partir du suivi hydrologique 2017-2018, montre que l'aléa «crue» $\left(2 \mathrm{~m}^{3} / \mathrm{s}\right.$ en pointe) est nul en dessous de $80 \mathrm{~mm}$ de cumul décadaire, mais de $37 \%$ audessus de ce seuil et de $66 \%$ au-dessus de $100 \mathrm{~mm}$. La figure $2 \mathrm{~b}$ signale le doublement des décades dépassant $80 \mathrm{~mm}$ de pluie cumulée pendant la période actuelle par rapport à la période sèche, donc un doublement de l'aléa de crues précoces. L'aléa de crues précoces dommageables aux cultures s'est donc aggravé par rapport à la période sèche et rejoint la valeur de la période historique.

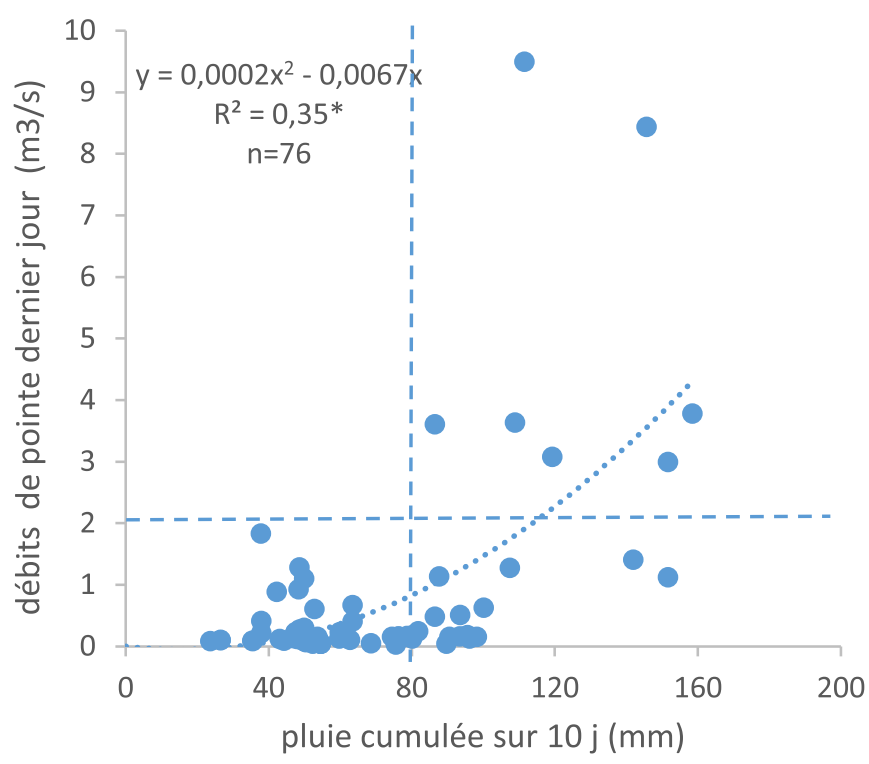

Fig. 3. Pointes de crue en juin-juillet en fonction du cumul décadaire de pluies. Les pointillés délimitent les crues jugées à risque $(>2 \mathrm{~m} / \mathrm{s}$ en pointe) et le seuil correspondant de $80 \mathrm{~mm} /$ décade.

Fig. 3. Floods in June-July in relation to cumulative decadal rain.

Cet accroissement de l'aléa de crues est confirmé par les paysans. Les crues endommageant cultures et ouvrages seraient en augmentation. Sur 14 parcelles étudiées en 2017 , $15 \%$ avaient été détruites par les crues en 2016. Des crues inondant le bas-fond sur toute sa largeur (environ $200 \mathrm{~m}$ ), avec une hauteur atteignant $50 \mathrm{~cm}$ au centre, se sont produites une fois en 2017 et trois fois en 2018 (Fig. 4b). Deux ont submergé le riz au stade végétatif. Enfin, 5 inondations durables de plus de $5 \mathrm{~cm}$ d'eau ont été notées en 2018, contre aucune en 2017 (Fig. 4b, mesures « externes »).

L'importance de la variation interannuelle du rendement (-34 \% en 2018 par rapport à 2017, pourtant concerné par une petite sécheresse en septembre) illustre la forte exposition de cette riziculture de bas-fond aux crues, même dans les contextes aménagés par canaux de drainage qui ne suppriment pas les crues. Les composantes déterminées précocement (biomasse, densité de panicules, densité de touffes, nombres de grains par panicule) ont fortement chuté en 2018 (Tab. 1). Les trois grandes crues des deux premiers mois auraient donc fortement affecté la production.

Enfin, il existe des périodes de saturation du sol en eau indépendantes des inondations (pic piézométrique de pression de la nappe décalé en septembre), qui transforment le bas-fond en source, comme en 2018, la nappe de bas-fond étant suralimentée par les nappes de versant (Fig. 4b). L'oxygène du sol peut alors faire défaut et pourrait aggraver l'aléa de toxicité ferreuse que l'on observe par endroits. Mais de tels symptômes n'ont pas été observés plus souvent à cette période, mais plutôt des symptômes de parasitisme (Ramphicarpa, et galles de cécidomyies), qui pourraient être les symptômes indirects d'un mauvais état physiologique du riz.

Le tableau 1 montre finalement la relative modestie des rendements moyens en riz de bas-fond ( $2 \mathrm{t} / \mathrm{ha}$ sur deux ans, toutes situations et années confondues), comparé au maximum observé de $6 \mathrm{t} /$ ha et leur grande variabilité $(\mathrm{CV}=63 \%)$. 

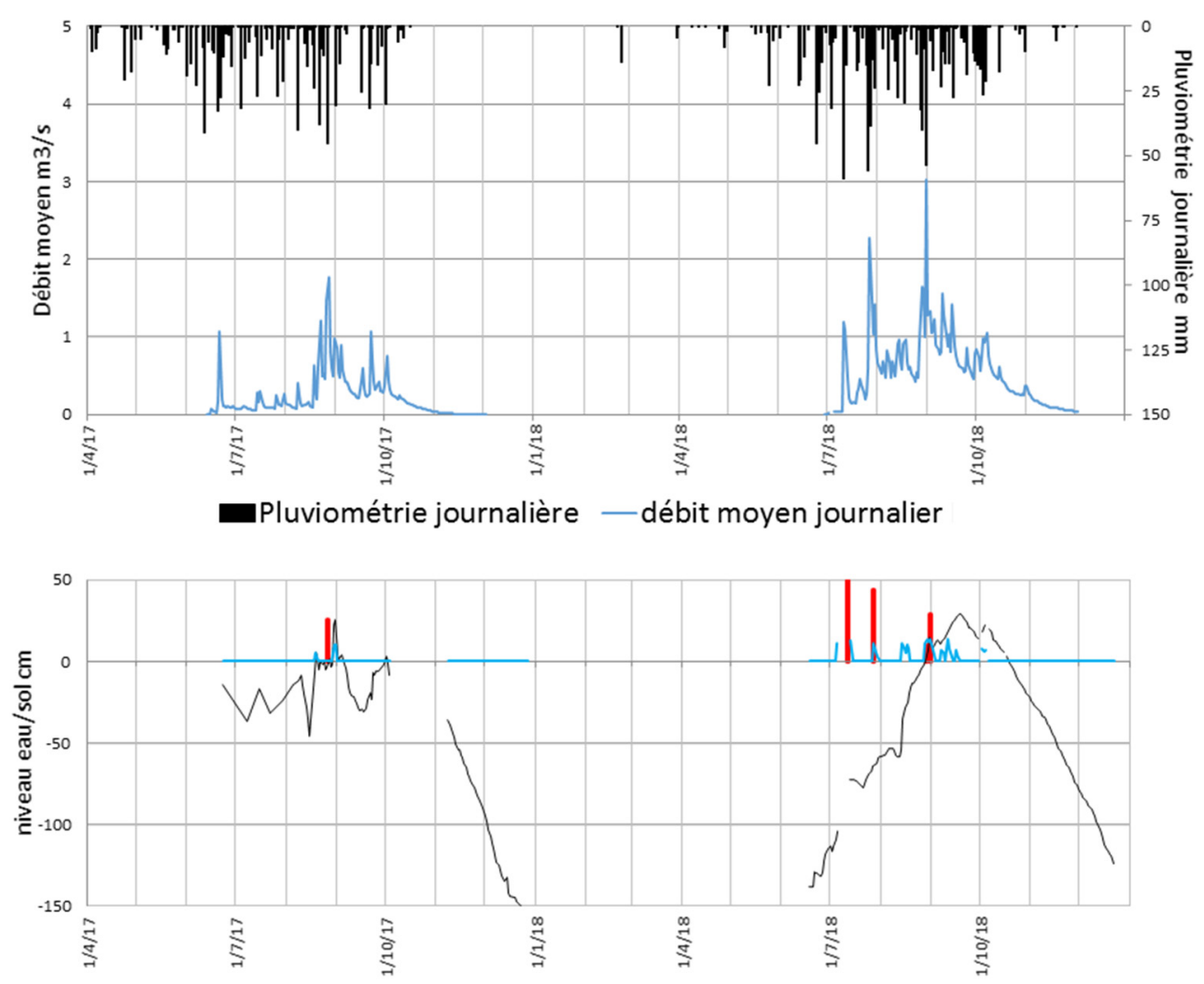

pics de crue $(\mathrm{cm})$ — piézomètre 22 mesure interne —mesure externe

Fig. 4. Pluviométrie, hydrométrie en 2017-2018 (4a en haut) et piézométrie journalière hors crue d'une section «non aménagée» (mesure externe de la lame d'eau sur le tube d'accès et niveau piézométrique interne) ( $4 \mathrm{~b}$ en bas).

Fig. 4. Rain, water flow and piezometry in an "unmanaged" section (external and internal measurement).

Tableau 1. Composantes du rendement parcellaire «riz de bas-fonds» (conditions aménagées ou non) deux années de suite, à Lofing. La différence entre années est significative (en gras) quand $p<0,05$ (test $T$ ).

Table 1. Yield components at plot scale (lowland rice).

\begin{tabular}{|c|c|c|c|c|c|}
\hline & Moyenne & $\mathrm{CV} \%$ & 2017 & 2018 & $p$-values $2018-2017$ ( $T$-test) \\
\hline Rendement MS paille (t/ha) & 2,5 & 60 & 3,9 & 1,8 & $\mathbf{0 , 0 0}$ \\
\hline Taux de touffes vides $(\%)$ & 6,6 & 85 & 3,1 & 8,4 & $\mathbf{0 , 0 0}$ \\
\hline$N$ grains/panicule & 78,0 & 35 & 92,7 & 71,5 & $\mathbf{0 , 0 0}$ \\
\hline Poids d'une panicule utile sèche $(\mathrm{g})$ & 1,72 & 32 & 1,85 & 1,65 & 0,13 \\
\hline Taux de panicules inutiles $(\%)$ & 10,4 & 68 & 10,5 & 10,3 & 0,92 \\
\hline Taux de grains vides $(\%)$ & 8,1 & 153 & 24,3 & 0,3 & $\mathbf{0 , 0 0}$ \\
\hline$N$ parcelles & 80,0 & & 26,0 & 54,0 & \\
\hline
\end{tabular}

3.2 Fonctions anti-aléatoires de la variété des usages 3.2.1 Un paysage changeant, mais témoin d'anciennes pratiques anti-risques

Encore forestier au $\mathrm{xIX}^{\mathrm{e}}$ siècle, le bas-fond a été progressivement défriché. Les résidences yirs entourées de champs de village se situent sur les bas de pente à proximité du bas-fond et de l'eau. La pêche et la cueillette d'herbes comestibles (Cassia, Corchorus...) et de fruits et de fourrages arboricoles (Ficus, Diospyros, Syzygium, Vitex, Cordia, Acacia...) dans le bas-fond réduisait les risques de famine pendant la soudure après les mauvaises années. 
Outre un filet d'eau permanent jusqu'en février, des mares et des puisards peu profonds permettaient d'accéder à l'eau jusqu'en juin, date de reprise d'un flux discontinu du marigot.

Le bas-fond a particulièrement été mis en valeur sous forme de buttes de cultures associées pendant la phase sèche 1971-1990. Cette fonction vivrière de soudure (maïs et aubergine récoltées en août, tubercules Coleus) exploitant la fonction sécuritaire de l'agro-diversité, ainsi que la cueillette persistante d'herbes comestibles, témoignent de la fonction de sécurité alimentaire qu'avait et qu'a encore le bas-fond pour une partie de la société locale.

En saison sèche, les résidus de récolte, les buttes en jachère à graminées pérennes et la présence de mares et d'arbres à fourrage comme les Ficus faisaient des bas-fonds un espace idéal pour l'élevage local (caprins et bovins) et pour le passage de transhumants.

Avec la raréfaction des sécheresses (années 1990) et l'augmentation du maïs pluvial en rotation avec le cotonnier, grâce à la culture attelée, les engrais «coton» et les nouvelles variétés de maïs introduites par l'encadrement technique, on assiste à un abandon partiel des buttes de maïs pénibles à entretenir. Un problème de divagation des bœufs (départ des gardiens Peuls après un conflit) accélère cette déprise. On assiste dans les années 2000 à une réduction du cheptel du village, due à la fois aux problèmes de gardiennage, de maladies du bétail, et de changements dans les pratiques de dot. Même les attelages se raréfient.

En 2013, Lofing bénéficie d'un aménagement rizicole pilote de la Fondation Dreyer (Fig. 5a, zone aval). Il s'agit d'un parcellaire de casiers traversé de trois canaux de drainage parallèles issus de trois déversoirs aménagés sur une digue transversale. Il permet d'accroître le domaine rizicole aux dépens de la zone pastorale devenue moins utile. Les arbres y ont été supprimés. La mise en valeur est individuelle $(0,10$ ha à 0,25 ha par personne attributaire), ce qui permet à certaines exploitations familiales de cumuler les parcelles. Des extensions ont été réalisées en 2015 et 2016.

Avec une mine d'or voisine apparue en mars 2018, les puisards du bas-fond sont mis à contribution pour la vente d'eau, et la main-d'œuvre disponible pour l'agriculture baisse fortement, le riz s'en ressentant particulièrement. Pour $\mathrm{y}$ pallier, en 2019, la Fondation Dreyer expérimente une nouvelle organisation de la production, en blocs mécanisés en régie, avec entretien du riz par groupes de membres de la nouvelle coopérative de riz de Lofing.

\subsubsection{L'aménagement multifonctionnel du bas-fond}

L'analyse spatiale (Fig. 5b) des sections «non aménagées » encore majoritaires montre en réalité de multiples formes d'aménagement de l'espace, des sols et de l'eau, individuels et collectifs. Le bas-fond «non aménagé » du quartier ZangoliHumboyir se partage à égalité entre :

- des pâturages collectifs et des mares au niveau du lit inondable du bas-fond, trop risqué pour les cultures. Le bétail est attaché à des piquets par des cordes («pachonage $\gg$ );

- des réseaux de buttes et plateformes vivrières occupant essentiellement les bandes latérales, dont un tiers est en jachère ;
- des parcelles labourées de riz à plat, extensives (pas de fertilisants, variétés locales), avec de petits aménagements de l'eau (digues de protection, fossé central, casiers à diguettes, batardeaux en terre);

- des plantations individuelles de teck sur les franges et une plantation villageoise.

Dans les zones riches en eau, des jardins avec puisards, parfois collectifs, sont entourés de digues plantées d'arbres, de fossés de ceinture qui protègent des crues et servent de base à un muret de protection en saison sèche. L'usage des mares est partagé entre jeunes jardiniers, éleveurs pour l'abreuvement et pêcheuses.

Avec l'urbanisation de Dano, ce maraîchage de saison sèche s'accroît dans les sections du bas-fond bénéficiant d'une nappe pérenne, soutenu par de nouveaux jardins grillagés et des puits busés financés par des Organisations non gouvernementales. Cette production de contre-saison répond aux opportunités du marché, mais aussi à la recherche de compléments de revenus face aux résultats variables de la saison des pluies.

\subsubsection{Les institutions de régulation locales et introduites}

Des institutions régulent les usages du bas-fond, conférant sécurité foncière à certains groupes et accès aux ressources selon une hiérarchie d'acteurs : le chef de terre villageois, les clans, les yirs jusqu'aux exploitations familiales et leurs individus. Les calendriers d'utilisation des mares sont convenus après négociation entre les parties, puis entérinés et proclamés par le chef de terre. Un interdit de consommation du crocodile au sein d'un des clans fondateurs de Lofing a favorisé la présence de ces sauriens au centre du terroir, qui garantissent aux yeux des habitants la pérennité de l'eau, l'identité de ce clan, et au-delà celle du village, conférant ici aussi un sentiment sécurisant.

Naturellement, la sécurité foncière reste inégale. Chaque clan fondateur dispose de droits fonciers sur un grand tronçon de bas-fond. Les yirs disposaient de droits d'appropriation sur des parcelles. Les membres de clans installés plus récemment sont donc moins favorisés. Ils peuvent néanmoins bénéficier de prêts de terres précaires, de même que les femmes lorsqu'elles veulent produire à titre individuel.

Aussi ces deux dernières catégories sont-elles intéressées par les aménagements exogènes (jardins maraîchers, jardins «nutritifs»-moringa, baobab- et périmètres rizicoles) susceptibles de leur attribuer durablement des parcelles de bas-fonds ou irrigables. Mais cette durabilité d'accès reste doublement incertaine. D'une part, les yirs, possesseurs coutumiers, pourraient se raviser avec le temps pour installer des héritiers. D'autre part, ces terres désormais dotées d'investissements collectifs dans un cadre coopératif, privé ou étatique sont aussi susceptibles d'être l'objet de réformes organisationnelles remettant tout en question, comme celle opérée par la Fondation Dreyer en 2019 avec le consentement de la nouvelle coopérative : réorganisation totale en «blocs coopératifs mécanisés en régie », dans l'espoir d'accroître le taux d'emblavement et le rendement en paddy.

Les filières de commercialisation se sont aussi diversifiées, avec le montage d'une rizerie à Dano par la Fondation Dreyer et d'une commercialisation directe vers celle-ci (groupage au 


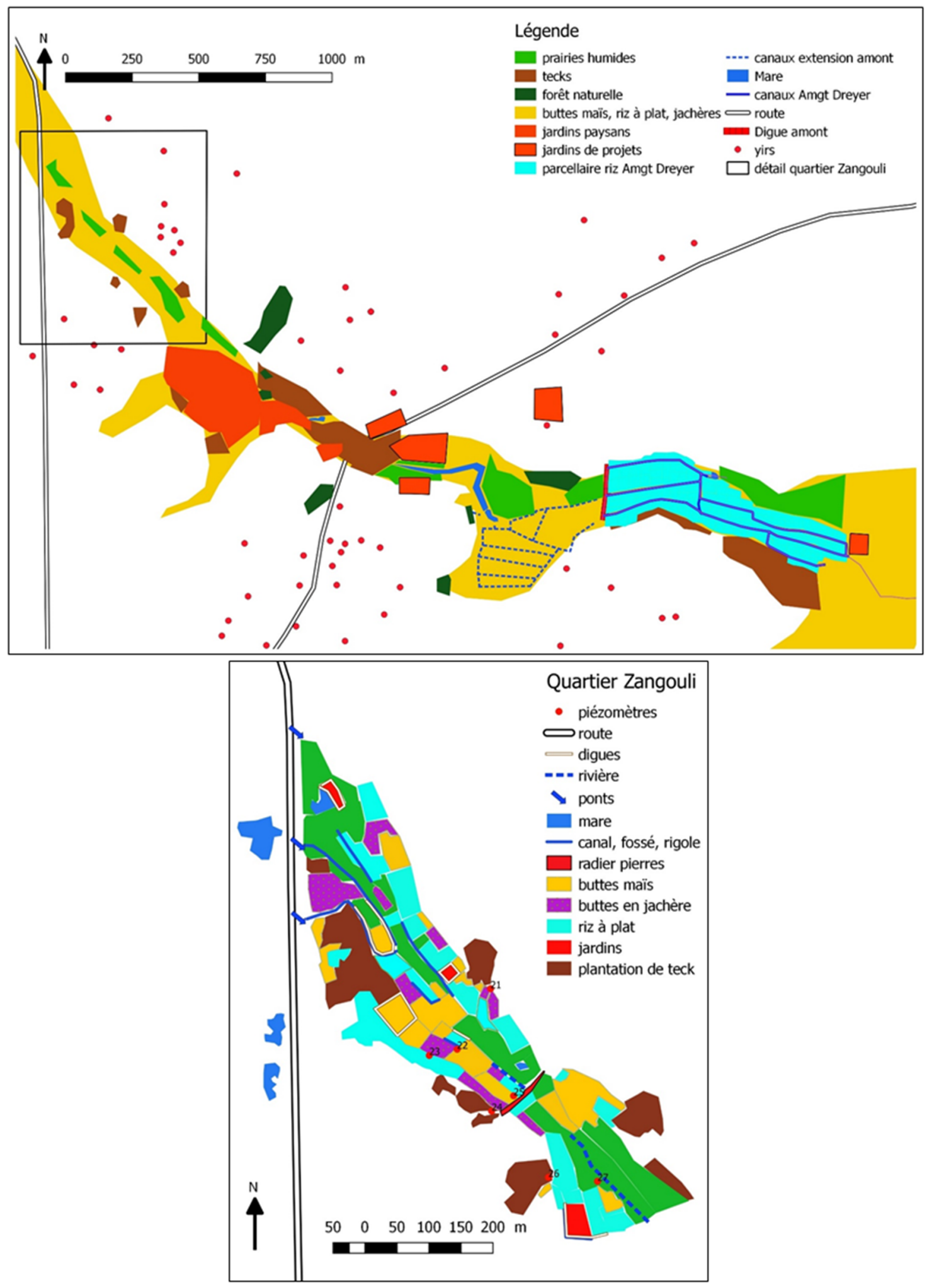

Fig. 5. Usages des bas-fonds de Lofing (5a) et détail de la section ouest «non aménagée » de Zangoli (5b).

Fig. 5. Land uses of Lofing valley and details of unmanaged west section. 
Tableau 2. Typologie des exploitations de Lofing.

Table 2. Farm typology of Lofing.

\begin{tabular}{|c|c|c|c|c|}
\hline Type d'exploitations & 1 & 2 & 3 & 4 \\
\hline Nombre dans échantillon & 4 & 5 & 5 & 4 \\
\hline Actifs familiaux permanents & 6 à 10 & 4 à 5 & 3 à 4 & 1 à 2 \\
\hline Superficie cultivée (ha) & 7 à 10 & 3 à 7 & 1,5 à 3,5 & 0,3 à 1,5 \\
\hline Niveau équipement & Culture attelée & Pulvérisateur manuel & $\mathrm{Nul}$ & $\mathrm{Nul}$ \\
\hline Cheptel (UGB) & 5 & 2 à 5 & 0 à 2 & 0 à 2 \\
\hline Budget intrants (Fcfa/campagne) & $>100000$ & 10000 à 100000 & $<10000$ & $<10000$ \\
\hline Budget main-d'œuvre (Fcfa/campagne) & $>100000$ & 10000 à 100000 & $<10000$ & 0 \\
\hline Part du riz dans la production commercialisable (\%) & 3 & 7 & 17 & 45 \\
\hline Part jardin dans la production commercialisable (\%) & 10 & 11 & 0 & 0 \\
\hline
\end{tabular}

Note: Les «résidents» sont un indice des besoins familiaux (besoins individuels pondérés par l'âge, adulte $=1$ ). Les «actifs » sont aussi un indice de main-d'œuvre familiale pondérée par l'âge, adulte $=1$ ).

niveau du groupement de riz de Lofing jusqu'en 2018). Mais une telle filière directe associant fourniture d'intrants et commercialisation, si elle sécurise les débouchés, réduit aussi les marges de manœuvre individuelles en imposant une variété unique, un prix et une date d'achat.

Ainsi, tant sur le plan physique que social ou économique, le bas-fond de Lofing reste marqué par une diversité d'usages, de règles, de types d'aménagements, et par des mutations révélant que de nouvelles fonctions productives et plus spécialisées lui sont assignées par les partenaires aménageurs : riz et maraîchage intensif dans des conditions d'un côté moins risquées grâce à des aménagements lourds (grillages, digues, canaux), aux organisations de producteurs et aux filières dédiées, mais d'un autre côté plus incertaines quant aux droits d'accès, marges de manœuvres individuelles et nouveaux risques.

\subsection{Place des bas-fonds dans la réduction des vulnérabilités familiales et individuelles}

Le tableau 2 résume les résultats de l'enquête «exploitations », sous forme d'une typologie de structure.

La typologie de structure réalisée sur l'échantillon restant correspond essentiellement à la taille de l'exploitation, car la surface par actif reste peu variable, à l'exception des plus grandes exploitations équipées en attelage (type 1). Une économie d'échelle se manifeste puisqu'elles seules parviennent à investir en matériel, en intrants, et en main-d'œuvre salariée, facilitant à la fois l'extension des surfaces et l'intensification culturale. La part du bas-fond dans la surface cultivée est toujours faible, mais augmente chez les plus petites exploitations (types 3 et 4), montrant l'importance du bas-fond pour les pauvres. Les exploitations de veuves constituent le groupe 4.

Dans les grandes exploitations, la place de la riziculture dans le travail est minoritaire, en raison à la fois du caractère mineur de l'espace de bas-fond ( $7 \%$ du finage) et de cette

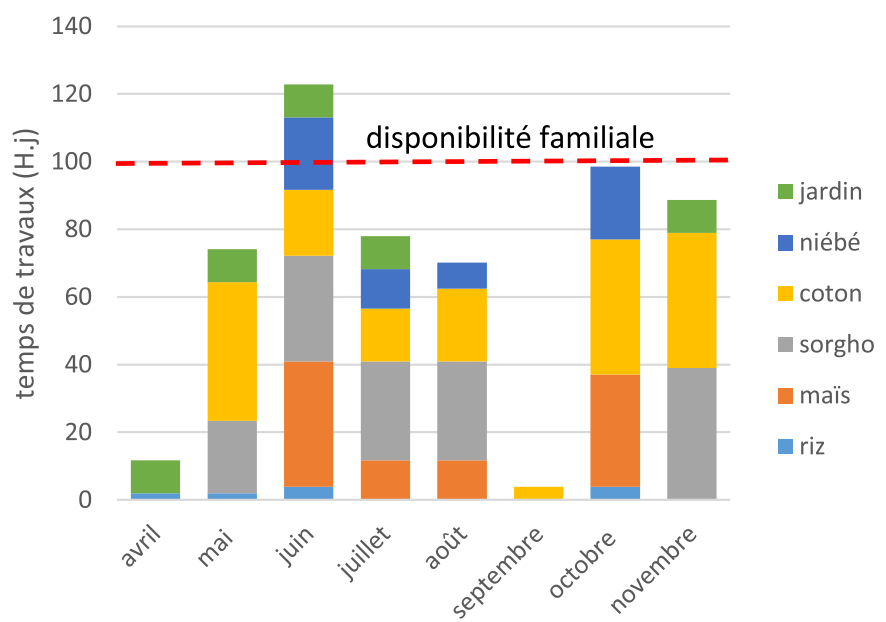

Fig. 6. Insertion du riz dans le calendrier de travail (type $2 ; 5,4$ ha; 100 homme $\times$ jour/mois disponibles).

Fig. 6. Place of rice in the work schedule (case of a middle farm).

culture (5 à $10 \%$ de la surface cultivée de l'exploitation). Néanmoins, si les premières averses sont précoces, elles permettent de commencer des travaux de piochage libérant du temps pour les autres cultures. Le mois de juin étant particulièrement saturé en termes de travail (Fig. 6), la main-d'œuvre rare et les adventices vivaces (Oryza longistaminata), le recours aux herbicides à haute dose est de plus en plus systématique, impliquant des risques environnementaux, mais aussi des symptômes fréquents de toxicité sur le riz.

La part du riz dans la consommation céréalière (fréquence de consommation par semaine) augmente, puisqu'elle atteint même $14 \%$ dans les plus grosses exploitations (signe d'aisance) et $7 \%$ dans les plus pauvres. Pour ces dernières, la riziculture est un facteur de diversification et d'accès aux terres, donc de sécurité alimentaire. 
La part du riz dans la valeur de production commercialisable des petites exploitations (notamment les veuves) est très importante $(45 \%)$ : c'est donc à la fois une production commerciale et une production vivrière pour ces dernières.

Les jardins apportent aussi des revenus consistants aux moyennes et grandes exploitations $(10 \%$ de la production commercialisable) et aux jeunes, à titre individuel. Faute d'accès aux terres, ils s'éloignent de l'agriculture, séduits par l'orpaillage ou les migrations, des options très risquées et qui réduisent la main-d'œuvre agricole. Certains pratiquent néanmoins le maraîchage en début de saison sèche.

Toutes les exploitations sont donc concernées par l'usage des bas-fonds, particulièrement les plus petites, et toutes les classes d'individus, particulièrement les plus vulnérables.

\section{Discussion et conclusion}

Les bas-fonds focalisent des enjeux importants pour la sécurité alimentaire et le revenu, à travers le maraîchage et le riz, mais aussi du fait de leur rôle pour l'élevage, la pêche et la production de bois de teck. Il subsiste des systèmes de culture et des aménagements paysans complexes, que Pradeau (1970) avait déjà mis en évidence dans le terroir Dagara de Kokolibou, dont le bas-fond était exploité en parcelles de riz à plat et buttes vivrières dans les années 1960. La multifonctionnalité du basfond s'inscrit dans un système agraire qui reste encore agrosylvopastoral, même si la part pastorale a fortement diminué.

Dans une zone rurale dense comme Dano, de nombreuses familles de petite taille sont vulnérables. Les veuves notamment se retrouvent chefs de petites exploitations avec un faible accès au capital et à la main-d'œuvre. Les jeunes sont aussi une catégorie marquée par l'exposition aux risques. Cette vulnérabilité sociale a augmenté depuis que les grands yirs Dagara solidaires ont fait place à des familles plus petites, moins bien dotées et affaiblies (Somé, 1990). Cette étude de cas confirme donc l'importance anti-aléatoire et multifonctionnelle des bas-fonds dans les zones soudaniennes à population dense. Elle identifie les nouveaux risques qui émergent du climat et des politiques actuelles, notamment une augmentation des évènements climatiques extrêmes (sécheresse et crues) en début de saison, une insécurité foncière croissante et des risques environnementaux.

Le rendement rizicole moyen reste modeste, du fait des contraintes, du caractère secondaire de ces productions et des nouveaux risques hydro-climatiques. La variabilité des rendements (de 0,4 à $6 \mathrm{t} / \mathrm{ha}$ ) exprime des tensions entre un potentiel satisfaisant et des contraintes sévères. À la pression des compétiteurs (mauvaises herbes, insectes) s'ajoutent les contraintes hydriques, crues, submersions, érosion (en 2018) et sécheresses (en 2017). Les crues affectent le riz via la perte d'azote du sol, les pertes d'engrais, l'état physiologique dégradé après submersion, les arrachages de pieds, l'érosion de surface et les ruptures de digues. Enfin, les pratiques restent marquées par la diversité, entre intensivité et extensivité selon la place et le rôle occupés par cette culture pour les individus et les exploitations. Ces limitations devraient freiner les ambitions productives des projets rizicoles et les inciter à réduire les principaux facteurs limitants (sensibilité variétale aux submersions, gestion de l'enherbement plus efficace et moins risquée pour l'environnement, approvisionnements en intrants sans retards, renforcement des digues et des déversoirs). Quant au taux d'emblavement des périmètres, il dépend surtout des motivations conjoncturelles, des coûts d'opportunité de la main-d'œuvre et du prix du paddy de l'année passée, comparé à celui du coton-graine connu d'avance.

Ces difficultés, d'une part, et ces fonctions socioéconomiques anti-aléatoires, d'autre part, nécessitent donc des précautions en cas d'aménagement rizicole lourd, notamment de limiter l'espace aménagé par rapport à l'espace disponible, de vérifier l'absence d'usage ou d'aménagements antérieurs, et de sécuriser l'accès aux terres attribuées tant qu'elles sont exploitées. Mais ces limites produiront ellesmêmes de nouvelles difficultés quant au partage de l'accès aux terres aménagées entre les groupes, faisant des «gagnants » et des «perdants». Une approche intégrée de l'aménagement du bas-fond dans son terroir serait donc nécessaire, rejoignant les préconisations de Rodenburg et al. (2013).

L'intérêt de cette étude est aussi son caractère transdisciplinaire. Les approches de l'adaptation au changement du climat mettent en avant la sécurité alimentaire (Brown et Crawford, 2008), mais de façon parfois réductrice, sans toujours replacer cette menace dans une hiérarchie de problèmes, comme la concurrence entre spéculations dans le calendrier de travail (coton vs. riz), la petitesse des parcelles qui démotive les plus nantis en moyens, et la valorisation économique du riz encore peu attractive en contexte de maind'œuvre disputée. Face aux aléas climatiques croissants, il convient de poursuivre la diversification des modes d'installation des cultures, des aménagements, des systèmes de culture, des qualités recherchées, bref d'encourager l'innovation.

Il est pourtant difficile de généraliser cette étude de cas. En effet, la littérature insiste fréquemment sur la diversité des situations de bas-fonds et des dynamiques agricoles (Raunet, 1985 ; Lavigne-Delville et Boucher, 1998). Une étude en cours sur une dizaine de bas-fonds (diagnostic participatif) et d'évaluation d'autres systèmes d'aménagement comme les « diguettes en courbe de niveau » (DCN) permettra d'aller plus loin dans l'évaluation des dynamiques d'aménagement à l'aune des fonctions productives, environnementales et sociales des bas-fonds.

Remerciements. Cette recherche est redevable au Ministère français des Affaires étrangères pour son financement du programme FSP AGRICORA Generia, à la population de Lofing (Dano) et à ses appuis régionaux (DRAAH, Fondation Dreyer). Un remerciement particulier aux relecteurs des Cahiers Agricultures.

\section{Références}

Audebert A. 2006. Diagnosis of risk and approaches to iron toxicity management in lowland rice farming. Iron toxicity in rice-based systems in West Africa. Cotonou (Benin): Africa Rice Center (WARDA), pp. 6-17.

Bainville S. 2017. Land rights issues in Africa: The contribution of agrarian systems research in Burkina Faso. The Journal of Peasant Studies 44(1): 261-285. 
Blaikie P, Cannon T, Davis I, Wisner B. 1994. At risk: Natural hazards, people's vulnerability, and disasters. Chapter eight: Earthquakes, volcanos and landslides. London (UK): Routledge, Taylor \& Francis Group.

Bossa AY. 2018. Gestion des nouveaux risques et des opportunités des terres inondables pour l'agriculture africaine. Projet Generia: Rapport d'étape, $32 \mathrm{p}$.

Brown O, Crawford A. 2008. Évaluation des conséquences des changements climatiques sur la sécurité en Afrique de l'Ouest. Étude de cas national du Ghana et du Burkina faso. Canada : IIED.

Dorée A. 2017. Gérer les nouveaux risques des bas-fonds soudaniens. Mémoire d'ingénieur agronome, option Resad. Montpellier (France) : Montpellier Supagro, IRD GRED, Cirad, Wascal, $84 \mathrm{p}$.

Drabo A. 2004. Situation de la filière riz au Burkina Faso, Observatoire national de la filière riz du Burkina Faso (ONRiz). Communication à l'Atelier PRIAF 10-14 mai 2004 à Bamako (Mali)

Eldin M, Milleville P. 1989. Le risque en agriculture. Paris (France): Orstom.

Franquin P. 1984. Agroclimatologie et agrométéorologie en zone tropicale sèche d'Afrique. Agronomie tropicale 39(4): 301-307.

Jamin JY, Windmeijer PN (eds). 1995. La mise en valeur durable des bas-fonds d'Afrique de l'Ouest. Premiers résultats du consortium bas-fonds, $118 \mathrm{p}$. Atelier annuel du consortium bas-fonds WARDA. 3, Bouaké, Côte d'Ivoire, 23-24 mars 1995.

Jouve P, Tallec M. 1994. Une méthode d'étude des systèmes agraires par l'analyse de la diversité et de la dynamique des agrosystèmes villageois. Cahiers de la recherche-développement 39: 43-59.

Lallau B. 2008. Les agriculteurs africains entre vulnérabilité et résilience. Pour une approche par les capabilités de la gestion des risques. Revue française de socio-économie 2008/1(1): 177-198. DOI: $10.3917 / \mathrm{rfse} .001 .0177$.

Lavigne-Delville P, Boucher L. 1998. Dynamiques paysannes de mise en culture des bas-fonds en Afrique de l'ouest forestière. In : Cheneau-Loquay A, Leplaideur A, eds. Les rizicultures de l'Afrique de l'Ouest. Actes du colloque international Cnrs-Cirad, Bordeaux, 5-7 avril 1995. Montpellier: Cnrs, Orstom, Cirad-ca, pp. $365-376$.

Leone F, De Richemond Meschinet N, Vinet F. 2010. Aléas naturels et gestion des risques. Paris (France) : PUF.

Lidon B. 1993. La mise en valeur agricole traditionnelle, fruit d'une longue expérience dans la gestion des risques. In: Albergel J, Lamachère JM, Lidon B, Mokadem A, Van Driel W, eds. Mise en valeur agricole des bas-fonds au Sahel. Typologie, fonctionnement hydrologique, potentialités agricoles. Ouagadougou (Burkina Faso): CIEH, 335 p.

Manzanilla DO, Paris TR, Vergara GV, Ismail AM, Pandey S, Labios $\mathrm{RV}$, et al. 2011. Submergence risks and farmers' preferences: Implications for breeding Sub1 rice in Southeast Asia. Agricultural Systems 104(4), 335-347.

Manzelli M, Fiorillo E, Bacci M, Tarchiani V. 2015. La riziculture de bas-fond au sud du Sénégal (Moyenne Casamance): enjeux et perspectives pour la pérennisation des actions de réhabilitation et de mise en valeur. Cahiers Agricultures 24(5): 301-312. DOI: 10.1684/agr.2015.0772.

Moody K. 1993. Weed control in wet seeded rice. Expl Agric 29(4): 393-403.

Pale S, Kiemde BJ, Da DE. 2016. Pratiques traditionnelles paysannes dans les bas-fonds agricoles à Dano au Burkina Faso. Rev Sc Env Univ Lomé 13: 122-136.

Pradeau C. 1970. Kokolibou (Haute-Volta) ou le pays Dagari à travers un terroir. Études rurales 37-39: 85-112.

Raunet M. 1985. Bas-fonds et riziculture en Afrique. Approche structurale comparative. L'Agronomie tropicale 40(3): 181-201.

Robin J. 2018. Les enjeux fonciers des aménagements de bas-fonds au sud-ouest du Burkina Faso: le cas de Lofing. Mémoire Master professionnel «Anthropologie et métiers du développement durable». Université Aix Marseille/IRD GRED, 81 p.

Rodenburg J, Zwart SJ, Kiepe P, Narteh LT, Dogbe W, Wopereis M. 2013. Sustainable rice production in African inland valleys: Seizing regional potentials through local approaches. Agricultural Systems 123(2014): 1-11.

Sakané N, Alvarez M, Becker M, Böhme B, Handa C, Kamiri HW, et al. 2011. Classification, characterisation, and use of small wetlands in East Africa. Wetlands 31: 1103-1116.

Savonnet G. 1976. Inégalités de développement et organisations sociales (exemples empruntés au sud-ouest de la Haute-Volta). Cah ORSTOM Ser Sci Hum 13(1): 23-40.

Serpantié G, Zombre P. 1994. Contraintes et potentialités des petits bas-fonds soudano-sahéliens vis-à-vis d'une riziculture sous aménagement d'étalement de crues. Expérience du programme R3S à Bidi (Nord Yatenga). Atelier national sur la riziculture et commission du programme riz Bobo-Dioulasso, 9-11 mai 1994.

Somé HP. 1990. Habitations et occupation du sol. Le yir et le village Dagara, l'exemple de Tobo. Cahiers d'Outre Mer 169: 77-85.

Yameogo PL. 2017. Managing seasonal soil nitrogen dynamics in inland valleys of the West African savanna zone. Ecology and Development Series. No. 102. Bonn: Eds ZEF, 99 p.

Citation de l'article : Serpantié G, Dorée A, Fusillier J-L, Moity-Maizi P, Lidon B, Douanio M, Sawadogo A, Bossa AY, Hounkpè J. 2019. Nouveaux risques dans les bas-fonds des terroirs soudaniens. Une étude de cas au Burkina Faso. Cah. Agric. 28 : 19. 